\title{
KAIZEN: UMA METODOLOGIA INOVADORA NA SIDERURGIA.
}

\section{RESUMO}

A racionalização do processo produtivo industrial, por meio da redução dos desperdícios e da modernização do processo produtivo, com a adoção do Sistema Toyota de Produção (Produção Enxuta), tem sido adotada por uma parte importante das indústrias no mundo. $\mathrm{O}$ objetivo deste artigo é descrever essa metodologia empregada pelo grupo de melhoria de fase intensiva adotado na Vallourec Tubos do Brasil S.A. Como resultado, verificou-se que o processo utilizado para a implantação de mudanças em curto prazo foi a semana intensiva, também conhecida como evento Kaizen na indústria automotiva.

Palavras-chaves: Metodologia Kaizen; Vallourec Tubos do Brasil S.A.; Sistema Toyota de Produção; Controle de Custos.

\section{KAIZEN: AN INNOVATIVE METHODOLOGY IN SIDERURGY.}

\begin{abstract}
The rationalization of the industrial production process, through the reduction of waste and the modernization of the production process, with the adoption of the Toyota Production System (Lean Production), has been adopted by an important part of the industries in the world. This article aim to describe this methodology used by the intensive phase improvement group adopted at Vallourec Tubos do Brasil S.A. As a result, the process used to implement short-term changes was the intensive week, also known as the Kaizen event in the automotive industry.
\end{abstract}

Keywords: Kaizen Methodology, Toyota Production System; Lean Production; Cost Control. 


\section{KAIZEN: UNA METODOLOGÍA INNOVADORA DE LA INDUSTRIA SIDERÚRGICA.}

\section{RESUMEN}

La racionalización del proceso de producción industrial, a través de la reducción de residuos y la modernización del proceso de producción, con la adopción del Sistema de Producción Toyota (Lean Production), ha sido adoptado por una gran parte de las industrias en el mundo. El propósito de este artículo es describir la metodología empleada por la fase intensiva del grupo de mejora aprobado en Vallourec Tubos Brasil SA Como resultado, se encontró que el proceso utilizado para los cambios a corto plazo en la implementación fue la semana intensiva, también conocido como el evento Kaizen en la industria automotriz.

Palabras clave: Metodología Kaizen; Vallourec Tubos Brasil. S.A.; Sistema de Producción de Toyota; Control de Costes.

Hericson Estanislau Prata ${ }^{1}$ Domingos Antônio Giroletti ${ }^{2}$

\footnotetext{
${ }^{1}$ Mestre em Admiistração pela Faculdade Pedro Leopoldo - FPL. Brasil. E-mail: hericson.prata@vallourec.com

${ }^{2}$ Doutor em Antropologia Social pelo Museu Nacional da Universidade Federal do Rio de Janeiro - PPGAS/UFRJ. Professor da Faculdade Pedro Leopoldo - FPL. Brasil. E-mail: domingosgiroletti@gmail.com
} 


\section{INTRODUÇÃO}

O Sistema Toyota de Produção (STP), também conhecido como produção enxuta ("lean production"), tem como objetivo produzir mais com menos tempo, estoques, recursos humanos, equipamentos, material e, ao mesmo tempo, atendendo às demandas dos clientes, segundo Dennis (2008).

O STP é bastante conhecido no setor automobilístico, e os ganhos obtidos pelas empresas desse setor são expressivos em termos de qualidade, segurança, produtividade e custos, na visão de Liker e Meier (2007). Os modos operantes das empresas lean começaram a fazer parte da realidade do setor siderúrgico, no qual esse tema é relativamente recente e, mesmo com iniciativas isoladas e aplicação de algumas ferramentas, percebem-se melhorias na produtividade.

A verdadeira eficiência do STP aparece quando a produção é capaz de eliminar os desperdícios, obtendo-se um índice de 100\% de trabalho. Ohno (1988) afirma que o passo preliminar para aplicação do STP na Toyota é identificar completamente os sete grandes desperdícios da linha de produção, sendo eles: movimento, espera, transporte, correção, excesso de processamento, estoque e excesso de produção.

$\mathrm{Na}$ visão de Slack, Chambers e Johnston (2009), as organizações precisam de direcionamento estratégico que contemplam suas atuais e futuras ações. Esse direcionamento é importante para a tomada de decisões, podendo ter efeito abrangente, definir a posição da organização e, por último, estabelecer os objetivos de longo prazo.

Com o objetivo de implementar as ações de melhorias, baseadas no direcionamento estratégico, será apresentada uma breve discussão sobre a metodologia do grupo de melhoria de fase intensiva (GMCi). A Vallourec Tubos do Brasil S.A (VBR) introduziu este novo formato de trabalho e tem tido bons resultados nas unidades de negócio.

O artigo está organizado em três partes, além da introdução. Na segunda, são discutidos os conceitos e o sistema de implantação da metodologia Kaizen. A terceira parte será dedicada à explicitação da metodologia de trabalho utilizada na Vollourec Tubos do Brasil, descrita em quatro fases. Na última parte, as considerações finais, serão avaliadas as vantagens auferidas pela VTB, ao adotar a metodologia descrita.

\section{CONCEITOS E IMPORTÂNCIA DO SISTEMA DE GERENCIAMENTO, EVENTO KAIZEN E PADRONIZAÇÃO}

O sucesso ou o fracasso do STP depende da atuação da liderança. Segundo Liker e Meier (2007), muitas tentativas de implantação desse sistema foram realizadas nas empresas por meio de contratação de consultores e implantação de eventos Kaizen, porém, com o passar do tempo, os programas foram esquecidos onde não houve envolvimento da liderança. Além disso, o envolvimento dos funcionários é o que move a produção lean e, por meio desse envolvimento, ocorre o desenvolvimento deles, podendo ter, por consequência, uma possível melhoria nos indicadores de performance das empresas (DENNIS, 2008).

Envolver os trabalhadores no planejamento de produção e na solução de problemas pode trazer um melhor resultado para a empresa. $\mathrm{O}$ engajamento dos empregados traz benefícios que podem melhorar as condições de trabalho e também reduzir ou facilitar a atividade. Entretanto, Dennis (2008) ressalta a importância da comunicação sobre a atividade, pois os trabalhadores precisam acreditar que as melhorias não implicarão demissões.

A liderança do STP tem um papel primordial no apoio da produção, na promoção do sistema e na liderança das mudanças. O papel do líder transcende a função de supervisor, pois ele conduz as mudanças, abrindo as portas para implantação de novos conceitos. Além disso, ele interpreta os objetivos da empresa e os desdobra em metas diárias para a produção, destacando as habilidades de liderança, de ensinar e de domínio das atividades. Segundo Liker e Meier (2007), esperase que o líder desempenhe as funções respeitando os aspectos de segurança, qualidade, produtividade e custo.

A Toyota utiliza o conceito da pirâmide invertida para a liderança, em que os líderes se encontram na base da pirâmide e não no topo. Assim, eles suportam diretamente a organização nas atividades que agregam valor, que são as linhas de produção. Liker e Meier (2007) destacam, ainda, algumas habilidades necessárias para a função de liderança: disposição e desejo de liderar, conhecimento do trabalho, responsabilidade, melhoria contínua e transferência dos conhecimentos adquiridos.

A solução de problemas específicos que buscam a melhoria contínua pode ser solucionada por meio de eventos Kaizen. Segundo Sharma e Moody (2003), uma característica desse evento é o trabalho em equipe, sendo fundamental o apoio da gerência, além dos recursos essenciais para execução da tarefa. O Kaizen é conhecido como um evento em que ocorrem mudanças substanciais, com resultados motivadores.

O evento Kaizen traz muitos benefícios, como o fortalecimento das habilidades de trabalho em equipe, liderança, pensamento objetivo e claro, e solução de problemas. Dennis (2008) afirma que os integrantes têm prazer com o trabalho, pois suas ideias são ouvidas pela organização. Quando um gerente tem algum trabalho para ser desenvolvido, cria-se um grupo de melhoria, e ele atua como um mediador na solução dos problemas.

Para registrar as atividades do evento Kaizen, usa-se o relatório A3, criado na década de 1960. O relatório tem somente uma página, sendo ele 
considerado a ferramenta de comunicação mais eficaz na Toyota, complementa Dennis (2008).

Liker e Meier (2007) mencionam que o evento Kaizen é dividido em cinco dias. O primeiro se refere ao treinamento sobre os conceitos básicos, para nivelar os integrantes do grupo. O segundo é composto por mais treinamentos e instruções para a visita à fábrica. Nesse dia, o foco do trabalho é medir, analisar, enxergar as oportunidades e priorizá-las. Os terceiro e quarto dias têm como objetivo a implantação das ideias levantadas. $\mathrm{O}$ autor descreve que não se pode corrigir os problemas em três dias, mas, com soluções simples, podem ser implantadas importantes ações. O quinto é dedicado à padronização, à apresentação, à visita ao local melhorado e, posteriormente, à confraternização pelos trabalhos executados.

No evento Kaizen, são realizadas análises para descobrir a causa raiz dos problemas. Kume (1993) descreve o procedimento para a construção do diagrama de causa e efeito, separando-o em cinco etapas: estabelecer a característica da qualidade; encontrar as possíveis causas; relacionar as causas com o efeito; estipular a importância dos fatores; e registrar as informações necessárias. Para o levantamento do maior número de causas, é necessária uma conversa aberta, usando a técnica de brainstorming, que significa um levantamento de ideias.

A apresentação dos resultados do Kaizen inclui a padronização e o roteiro das atividades desenvolvidas ao longo da semana. O grande desafio é passar para a direção da empresa a mensagem correta a respeito dos resultados atingidos em uma semana de trabalho, conforme mencionado por Liker e Meier (2007).

A padronização no STP representa a forma mais fácil, segura e eficaz para a execução de uma atividade. $\mathrm{O}$ trabalho padronizado traz vários benefícios, como: estabilidade dos processos que permite a repetição na elaboração dos produtos; clareza sobre o início e fim das atividades; aprendizagem organizacional; facilidade para auditorias; envolvimento com funcionários; e treinamentos dos funcionários (DENNIS, 2008). No próximo item, será apresentada a metodologia empregada no GMCi adotado na VBR.

\section{GRUPOS DE MELHORIA CONTÍNUA DE FASE INTENSIVA NA VBR}

A VBR tem trabalhado desde o ano $2000 \mathrm{com}$ a metodologia dos grupos de melhoria contínua (GMCs), segundo Kapferer e Breton (2009). Entretanto, com o objetivo de acelerar as economias, foi necessária a criação de uma nova modalidade de grupo de melhoria contínua, pois trabalhar com grupos que se reuniam uma vez por semana não gerava uma resposta rápida para atender às necessidades da demanda de mercado.

Segundo Sharma e Moody (2003), o Kaizen é conhecido como um evento em que ocorrem grandes mudanças com resultados motivadores em curto período. A VBR criou o GMCi em outubro de 2011, similar ao Kaizen. Entretanto, a empresa retirou da semana intensiva o treinamento e a levantamento das informações, pois ela entende que com um treinamento bem elaborado e uma boa base de dados, a fase intensiva poderá ser potencializada.

A FIGURA 1 apresenta as quatro fases para a formação do GMCi: a definição da equipe, escopo de trabalho e treinamento de nivelamento do grupo; a preparação; a semana intensiva; e, por último, a fase de acompanhamento e sustentação dos resultados. A VBR utiliza o DMAICS como método de solução de problemas. DMAICS é uma sigla que significa: "definir, medir, analisar, implementar, controlar e padronizar". A metodologia DMAICS é similar ao PCDA descrito por Campos (1992).

Figura 1 - Fases do grupo de melhoria contínua de fase intensiva

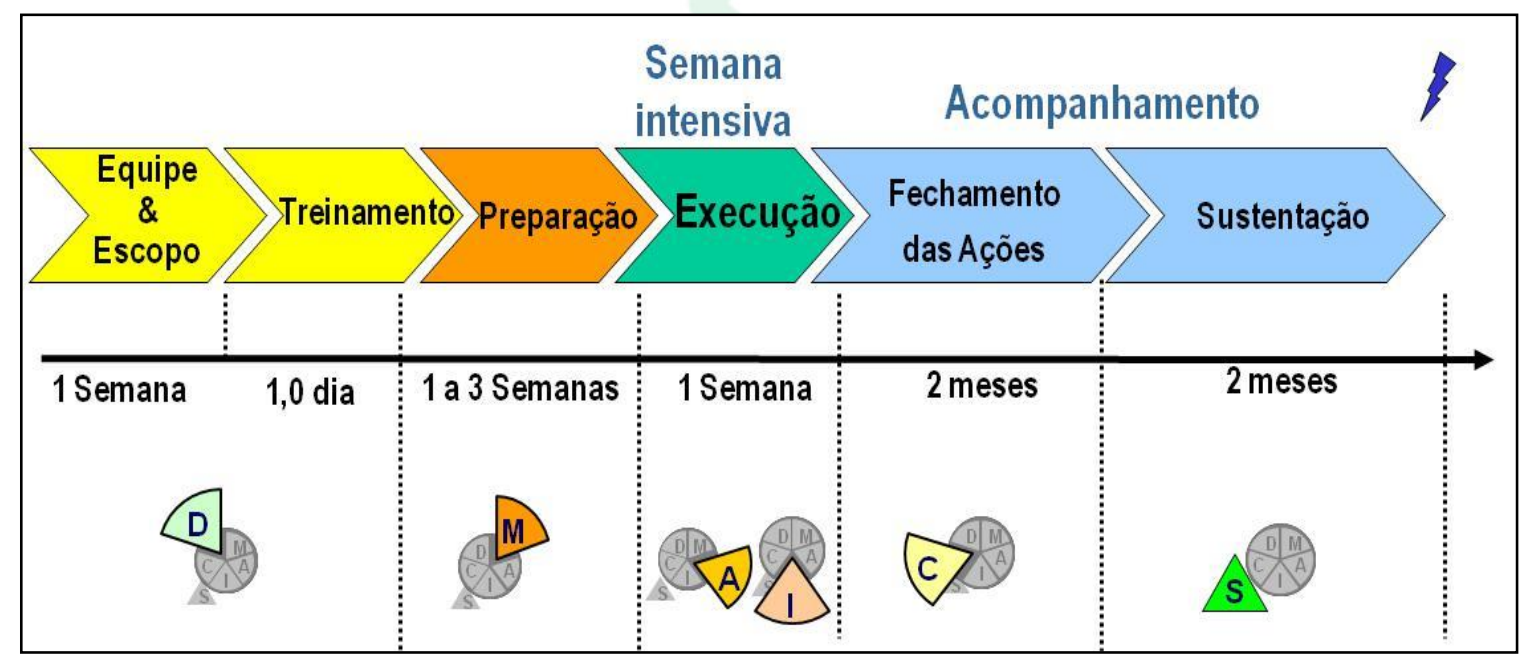

Fonte: VBR, 2012a. 
Todas as fases são importantes, entretanto a semana intensiva requer uma atenção especial, pois todos os membros da equipe deixam suas atividades para se dedicarem ao trabalho em grupo. O sucesso ou o fracasso do grupo de melhoria depende da atuação da liderança, segundo Liker e Meier (2007).

Em cada fase, a equipe de trabalho se reúne para preencher o A3, que é o relatório de uma página, conforme Dennis (2008). Por meio desse relatório, todos têm as informações necessárias sobre o andamento do projeto.

O formulário A3 da VBR possui nove itens, sendo eles: contexto, condições iniciais, objetivos e metas, análise, soluções propostas e plano de implementação, controle, padronização, acompanhamento e ganho apurado. No A3 também é identificado o método de solução de problemas na VBR, assim definido: contexto e condições iniciais pertencem à fase de definição e objetivos e metas, à de medição; análise é a designação da terceira; soluções propostas e plano indicam a quarta fase de implementação; acompanhamento relaciona-se à fase de controle e o restante, à de padronização.

\section{Fase 1: Definição da equipe, escopo de trabalho e treinamento de nivelamento do grupo}

Na VBR existe um comitê de governança para implantação do STP em cada unidade de negócio. Este comitê decide quais GMCis serão criados, baseado na aplicação de algumas ferramentas de análise do STP. Considera-se também a necessidade dos clientes internos ou externo, o alinhamento com a estratégia da empresa e a definição do assunto e perímetro, pois o objetivo é resolver problemas específicos através de soluções simples.

Conforme Liker e Meier (2007), o sucesso ou o fracasso do STP depende da atuação da liderança. Para a definição do líder do grupo, a VBR estabeleceu alguns critérios de seleção, sendo eles: proatividade, domínio da metodologia, comprometimento, bom relacionamento e experiência. Entretanto, é levado em consideração também, se o escolhido quer ser o líder do grupo.

Segundo Dennis (2008), os integrantes têm prazer com o trabalho porque suas ideias são ouvidas pela organização e, na equipe, possuem responsabilidades durante o trabalho. A equipe tem que dar suporte ao líder, manifestar sempre a opinião, dividir com o grupo todas as informações, não ter medo de errar e atuar com motivação.

Um ponto levado muito relevante é a disponibilidade do integrante, pois o trabalho requer dedicação exclusiva ao assunto trabalhado. Assim, a participação dos operadores é muito forte no $\mathrm{GMCi}$, evidenciando o conceito da pirâmide invertida citado por Liker e Meier (2007).

Definidos o escopo e a equipe, inicia-se o treinamento de nivelamento do grupo. Este treinamento tem duração de até oito horas e contempla alguns conceitos básicos do STP, ferramentas que poderão ser utilizadas e a metodologia do GMCi. As ferramentas podem ser: $5 \mathrm{~S}$, sistema de troca rápida de ferramentas, padronização, mapa de fluxo de valor, fluxograma de processo, diagrama de espaguete, diagrama de causa e efeito, matriz esforço e impacto, entre outros.

Após este treinamento, o grupo preenche a primeira parte do $\mathrm{A} 3$, que se refere aos campos do contexto atual e condições iniciais. No contexto é descrito a importância do trabalho e a aderência com a estratégia, enquanto nas condições iniciais são relatados os resultados atuais e os problemas enfrentados.

\section{Fase 2: Preparação}

A fase de preparação para a semana intensiva é marcada pelo levantamento das informações necessárias para se conhecer o estado atual. É importante também o preparo dos materiais de escritório tais como post-it, folhas em branco, canetas, cola, lápis, caneta, entre outros, pois são as ferramentas de trabalho do GMCi.

Kume (1993) menciona que são realizadas análises para descobrir a causa raiz dos problemas. $\mathrm{Na}$ VBR o estado atual pode ser definido usando técnicas de análise de dados históricos, mapeamento do fluxo de valor e mapa ou fluxograma de processo. A empresa trabalha também com sete ferramentas da qualidade visando ao entendimento da situação a ser trabalhada para encontrar a causa raiz dos problemas: banco de dados, gráficos, histograma, diagrama de Pareto, diagrama de causa e efeito, diagrama de correlação e carta de controle.

A empresa utiliza as informações levantadas e análises de forma bem visual e de fácil acesso, para facilitar a compreensão de todos. Verifica-se a confiabilidade dos dados, medindo o problema usando indicadores de performance, com o objetivo de se ter uma visão clara da situação analisada.

Nesta fase verifica-se a disponibilidade de toda a equipe para a semana intensiva, pois a participação em tempo integral é fundamental para o sucesso do grupo. Além disso, elabora-se um plano com os gestores sobre a participação deles na reunião de abertura, fechamento da semana e nos fechamentos diários.

O período de duração dessa fase prevê, em média, de uma a três semanas. Isso dependerá do assunto trabalhado e da frequência das reuniões. Na VBR é recomendado reunir-se quatro horas por semana.

$\mathrm{Na}$ fase da preparação, a equipe preenche o item três do formulário A3 que se refere aos objetivos e metas. Conhecendo a situação atual, é definido o que será feito, estabelecendo os limites do trabalho. Além disso, é importante traduzir os objetivos e metas em indicadores para serem acompanhados pelo grupo. 


\section{Fase 3: Fase intensiva}

Liker e Meier (2007) relatam que o evento Kaizen é dividido em cinco dias com dedicação exclusiva à solução dos problemas. Na VBR a fase intensiva do GMCi foi também dividida em cinco dias.

Segundo Sharma e Moody (2003), uma característica do evento Kaizen é o trabalho em equipe, sendo fundamental o apoio da gerência, além dos recursos essenciais para execução das tarefas diárias. A VBR priorizou a semana intensiva como o período para análise final, criação do plano de ação e implantação das ideias.

No QUADRO 1, são apresentadas as principais atividades desenvolvidas nos cinco dias da semana intensiva. Destacam-se a reunião de abertura, o fechamento diário com o gestor ou patrocinador do grupo, a grande ênfase na implantação de ideias simples, a visita à área modificada e, por último, a apresentação-aula sobre todas as fases anteriores e os cinco dias intensivos.

\begin{tabular}{|c|c|c|c|c|}
\hline $1^{\circ}$ dia & $2^{\circ}$ dia & $3^{\circ}$ dia & $4^{\circ}$ dia & $5^{\circ}$ dia \\
\hline Reunião de abertura & $\begin{array}{c}\text { Seleção da solução } \\
\text { para atingir a } \\
\text { situação futura }\end{array}$ & \multirow{3}{*}{$\begin{array}{l}\text { Implementação das } \\
\text { soluções }\end{array}$} & \multirow{3}{*}{$\begin{array}{c}\text { Implementação das } \\
\text { soluções }\end{array}$} & \multirow{2}{*}{$\begin{array}{c}\text { Padronizar } \\
\text { principais ações }\end{array}$} \\
\hline $\begin{array}{c}\text { Realizar análise de } \\
\text { causa }\end{array}$ & \multirow{2}{*}{$\begin{array}{l}\text { Implementação das } \\
\text { soluções }\end{array}$} & & & \\
\hline $\begin{array}{l}\text { Levantamento de } \\
\text { ideias para definir } \\
\text { situação futura }\end{array}$ & & & & Visita ao Gemba \\
\hline $\begin{array}{l}\text { Reunião com o } \\
\text { gestor }\end{array}$ & $\begin{array}{l}\text { Reunião com o } \\
\text { gestor }\end{array}$ & $\begin{array}{l}\text { Reunião com o } \\
\text { gestor }\end{array}$ & $\begin{array}{l}\text { Reunião com o } \\
\text { gestor }\end{array}$ & $\begin{array}{l}\text { Apresentação final } \\
\text { dos trabalhos }\end{array}$ \\
\hline
\end{tabular}

Quadro 1 - Principais atividades dos cinco dias da semana intensiva Fonte: VBR, 2012b.

O primeiro dia da semana intensiva é marcado pela reunião de abertura, realizada entre os gestores e os integrantes do grupo de trabalho, pela análise das causas, levantamento de ideias e a reunião de fechamento do dia. A VBR estabeleceu alguns mandamentos da semana intensiva, tais como: evitar atitudes de desculpas e justificativas, gastar energia na busca de soluções, fazer as ações imediatamente, aproveitar os problemas e dificuldades para encontrar as melhores ideias e soluções, buscar o bom resultado não se preocupando com a perfeição, substituir trabalhos e movimentos inúteis pelos úteis e lucrativos e quebrar paradigmas não aceitando verdades como absolutas.

$\mathrm{Na}$ reunião de abertura o gestor reforça o compromisso do grupo com a identificação da causa raiz, o desenho da situação futura, a seleção de soluções simples, rápidas e com custos mínimos, a implantação das ideias e a padronização das principais ações. Como o grupo teve o treinamento de alinhamento na fase de preparação, iniciam-se as atividades analisando a situação atual e levantando ideias de melhoria, baseadas nas ferramentas do STP e de qualidade da VBR. Estas ideias, descritas em post-it, ficam dispostas em um quadro que traz as informações do problema levantado, da causa raiz identificada e da ação identificada.

No final do primeiro dia é realizada uma reunião de acompanhamento para comparar o planejamento estabelecido para o dia com o realizado. A participação dos gestores é muito importante, pois além de monitorar o andamento da semana, verificamse as necessidades de recursos adicionais para a sequência dos trabalhos. Conforme Liker e Meier (2007), o sucesso e/ou o fracasso do STP dependem da atuação da liderança.

O segundo dia da semana intensiva contempla a seleção da solução para atingir a situação futura e o início da implementação das ideias. Para esta seleção, utiliza-se uma matriz de priorização que leva em consideração o impacto ou ganho com a ação e a dificuldade de implementação.

$\mathrm{Na}$ figura 2 é apresentada a matriz de priorização utilizada na VBR. A matriz possui nove quadrantes. Os quadrantes de 1 a 4 possuem cores verde, pois representam ações que possuem maiores impactos e baixas dificuldades de implantação. Para estes quadrantes, o grupo possui uma meta de $70 \%$ para a implantação das ações durante a semana intensiva. Os quadrantes de 5 a 8 possuem cores laranja com prioridades mais baixas e o grupo pode implementá-las durante a semana intensiva. O quadrante 9 possui a cor vermelha que significa que o grupo não implantará as ações classificadas neste quadrante, porque requer um esforço elevado com baixo impacto. 
Figura 2 - Matriz de priorização

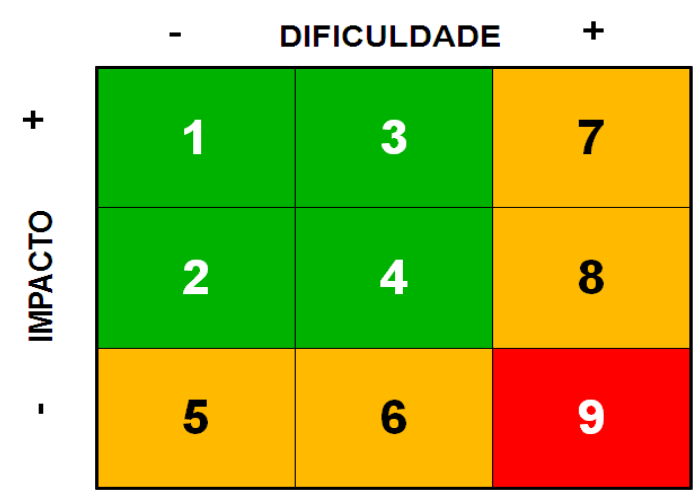

Fonte: VBR, 2012c.

Após a priorização, o grupo de trabalho elabora um plano de ação contendo, preferencialmente, as ideias dos quadrantes 1 ao 4, nomeando um responsável pela implantação da ação e o prazo de conclusão dentro da semana intensiva. Como o prazo máximo de implantação é de aproximadamente três dias, equipes de manutenção e operadores ficam à disposição para ajudar nessa tarefa.

A implantação das ideias é centralizada do segundo ao quarto dia. Isso requer um planejamento antecipado do grupo que ocorre na etapa da preparação, pois nessa fase os recursos precisam ser bem planejados, visando ao cumprimento da meta de $70 \%$ das ideias implantadas na semana intensiva.

No quarto dia ocorre a validação dos resultados de algumas ações. Essas ações precisam ser monitoradas e testadas pela equipe de operação para evitar um cancelamento posteriormente. Assim, após o teste na área, o grupo apresenta os resultados para validação dos gestores no final do dia.

O quinto dia é marcado pela padronização das principais ações, pela visita à área estudada com os gestores e o grupo, pela apresentação final e uma pequena celebração dos resultados alcançados. Silva (1996) menciona oito passos do método de solução de problemas e a padronização é parte integrante deste processo. Na VBR, a padronização é mais visual, com fotos ilustrando as atividades e com uma pequena descrição de cada etapa.

$\mathrm{Na}$ fase da semana intensiva a equipe preenche os itens 4 e 5 do formulário A3 que se referem às fases de análise, às soluções propostas e ao plano de implementação. $\mathrm{Na}$ análise é importante identificar a causa raiz do problema evidenciando as ferramentas utilizadas. Já nas soluções propostas e no plano, torna-se relevante mencionar como a solução tomada afeta o problema e qual foi o conjunto de ideias implementadas.

\section{Fase 4: Fechamento das ações e sustentação dos resultados}

Após a semana intensiva pode existir ações que não foram concluídas. Preferencialmente, elas deveriam ter sido concluídas naquele período. Entretanto, a VBR estabeleceu como regra o prazo de dois meses para fechamento das ações planejadas.

No período posterior à semana intensiva, parte do grupo se reúne uma vez por semana com o objetivo de acompanhar a conclusão das ações. Este grupo também certifica se os documentos e padrões elaborados foram devidamente atualizados no sistema integrado de gestão da VBR.

Finalizado o prazo de conclusão das ações, o grupo inicia a fase de monitoramento dos resultados. Assim, os resultados são acompanhados pelo mesmo período do fechamento das ações, ou seja, dois meses. Se os resultados estiverem dentro do previsto, o grupo é encerrado. Caso contrário o grupo se reúne para verificar os padrões e anomalias. O resultado desta análise é informado ao comitê de gerenciamento da unidade de negócio para a tomada de decisão sobre o destino do trabalho.

Nesta última fase, o grupo preenche os itens 6 ao 9 que se referem ao controle, padronização, acompanhamento e ganhos. Este formulário A3 é o documento oficial do GMCi onde são consultadas todas as informações sobre as etapas realizadas pela equipe de trabalho.

Com o objetivo de facilitar os trabalhos das equipes, a VBR criou um guia denominado "Check list para condução de grupos de fase intensiva". Este guia traz informações para as fases de preparação, execução e fechamento do grupo. Trata-se de lembretes importantes, tais como: reserva de agenda, apresentação do treinamento, documentação necessária, assuntos para abertura e fechamento da semana intensiva, reunião pós semana intensiva, entre outros.

A VBR faz uma verificação interna para acompanhar os grupos encerrados com o objetivo de 
identificar o melhor grupo da empresa. É avaliado o alinhamento do grupo com a estratégia, a documentação utilizada pelo grupo, a equipe de trabalho, a análise dos problemas, o levantamento da causa raiz, a priorização, o plano de implementação, resultados alcançados, padronização e a transferência de conhecimentos para outras áreas. No final de cada ano o melhor grupo é contemplado com uma viagem internacional.

\section{CONSIDERAÇÕES FINAIS}

A redução dos custos de produção e a eliminação dos desperdícios são metas cobiçadas pelas empresas, especialmente em época de crise. Produzir mais com menos recursos tem sido a tônica do momento, devido, principalmente, à concorrência acirrada, que cria uma necessidade de modernização cada vez mais forte. Essa necessidade de mudança gera também uma necessidade de análise do fluxo de valor. Com ações simples, rápidas e eficazes em termos de resultados, o Sistema Toyota de Produção analisa o fluxo de valor, com o objetivo de eliminar os desperdícios no processo de produção.

A implantação de forma eficiente da filosofia do STP na VBR, acompanhadas pelos comitês diretivos, tem sido uma das preocupações da empresa para o estabelecimento de um novo sistema de gerenciamento. Como este assunto é relativo novo no setor siderúrgico, apesar dessa metodologia ser muito difundida no setor automobilístico, a VBR não quer se impressionar com os bons resultados produtivos alcançados com a aplicação de algumas ferramentas, pois o foco é a implantação de uma nova forma de trabalho.

A implantação desse sistema de gerenciamento tem como objetivo aumentar a competitividade da VBR diante das demais empresas fabricantes de tubos, tendo em vista que o mercado está cada vez mais acirrado. Além disso, o aumento da competitividade pode evitar o ingresso de novos entrantes no mercado brasileiro.

A VBR adotou o GMCi como o motor da transformação. A adoção deste novo formato de grupo de melhoria provocou a quebra de um grande paradigma: a dedicação de cinco dias para fazer melhoria contínua. Anteriormente, o grupo de melhoria se reunia uma hora por semana continuamente. A partir do momento em que os empregados perceberam a força deste novo grupo na solução dos problemas, no senso de urgência e no comprometimento do grupo, essa nova modalidade de trabalho tornou- se uma realidade nas atividades da área de produção.

Outro paradigma quebrado foi com relação à chegada de mais uma ferramenta corporativa. Os envolvidos perceberam que o STP não é uma ferramenta que se aplica, é um sistema de gerenciamento que utiliza o GMCi para auxiliar na implantação de novas ideias.
Pode-se afirmar que, com a implantação do STP na VBR, utilizando GMCi foi possível reduzir os desperdícios da produção, aproveitando melhor os recursos existentes. Conforme Dennis (2008), o STP permite produzir mais utilizando menos tempo, estoques, recursos humanos, equipamentos e materiais e, principalmente, colocando o cliente como foco para estabelecer o ritmo da produção.

A VBR já implantou vários GMCis em várias unidades de negócio. Os resultados alcançados foram muito satisfatórios e a padronização da metodologia facilitou sua implantação, permitindo o desenvolvimento de vários multiplicadores dentro da empresa.

\section{REFERÊNCIAS}

Campos, Vicente Falconi. TQC: controle da qualidade total (no estilo japonês). (1992). 2. ed. Belo Horizonte: Fundação Christiano Ottoni.

Dennis, Pascal. Produção Lean simplificada. (2008). 2. ed. Porto Alegre: Bookman.

Kapferer, Patrícia; Breton, Tristan Gaston. (2009). Vallourec no centro da excelência: leader mondial. Paris: K.GB\&Co.

Kume, Hitoshi. (1993). Métodos estatísticos para melhoria da qualidade. São Paulo: Gente.

Liker, Jeffrey K.; Meier, David. (2007). O modelo Toyota: manual de aplicação: um guia prático para a implementação dos 4 PS da Toyota. Tradução de Lene Belon Ribeiro. Porto Alegre: Bookman.

Ohno, Taiichi. (1988). Toyota production system: beyond large-scale production. New York: Productivity Press.

Prata, Hericson Estanislau. (2013). Modernização produtiva da VALLOUREC: avaliação da implantação do Sistema Toyota de Produção. Dissertação de mestrado. Pedro Leopoldo: FPL.

Sharma, Anand; Moody, Patrícia E. (2003). A máquina perfeita: como vencer na nova economia produzindo com menos recursos. Tradução de Maria Lucia F. Leite Rosa; revisão técnica Carlos Louzada. São Paulo: Pearson Prentice Hall.

Slack, Nigel; Chambers, Stuart; Johnston, Robert. (2009). Administração da produção. Tradução de Henrique Luiz Corrêa. 3. ed. São Paulo: Atlas.

VBR. (2012). Tutorial de fase intensiva. Belo Horizonte: Vallourec, 2012a, b e c. Disponível em:

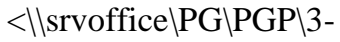
BibliotecatemáticalGMCI\GMCI Tutorial>. Acesso em: 4 ago. 2015. 